FORMATION Formation emploi

Revue française de sciences sociales

143 | Juillet-Septembre 2018

Le retour en formation : une vraie chance?

\title{
Présentation de l'ouvrage de N. Mohib et S. Guillon, La professionnalisation en débat : entre intentions et réalisations
}

Muriel Béasse

\section{(2) OpenEdition \\ Journals}

Édition électronique

URL : http://journals.openedition.org/formationemploi/6383

DOI : $10.4000 /$ formationemploi.6383

ISSN : 2107-0946

Éditeur

La Documentation française

Édition imprimée

Date de publication : 20 novembre 2018

Pagination : 221-224

ISSN : 0759-6340

\section{Référence électronique}

Muriel Béasse, «Présentation de l'ouvrage de N. Mohib et S. Guillon, La professionnalisation en débat: entre intentions et réalisations », Formation emploi [En ligne], 143 | Juillet-Septembre 2018, mis en ligne le 20 novembre 2018, consulté le 07 novembre 2020. URL : http://journals.openedition.org/

formationemploi/6383 ; DOI : https://doi.org/10.4000/formationemploi.6383 


\title{
Note de lecture
}

\section{Présentation de l'ouvrage de N. Mohib et S. Guillon, La professionnalisation en débat: entre intentions et réalisations}

\author{
Muriel Béasse \\ Doctorante, Laboratoire interuniversitaire des sciences de l'éducation \\ et de la communication, Lisec-EA2310
}

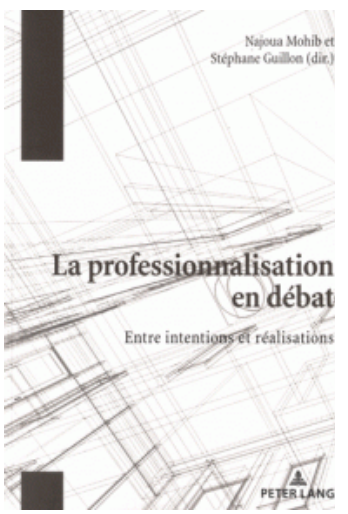

La professionnalisation est une notion équivoque qui s'impose dans diverses dimensions du monde professionnel et éducatif, sans toujours nous révéler ses ambiguïtés et ses enjeux. Mais de quoi parle-t-on vraiment quand on parle de professionnalisation ? Objectif politique prioritaire pour certains, le mouvement de professionnalisation est souvent associé aux valeurs néolibérales et envisagé comme un processus de formation et de validation de compétences.

Cet ouvrage collectif apporte un éclairage critique essentiel sur cette notion en vogue. Najoua Mohib et Stéphane Guillon, chercheurs en sciences de l'éducation à l'université de Strasbourg, ont rassemblé pas moins de quinze contributions d'auteurs sur la question de la professionnalisation, en l'abordant aussi bien du point de vue de la recherche et du milieu éducatif que de celui du monde professionnel.

\section{Les impacts spécifiques à chaque milieu professionnel}

Trois grandes parties composent cet ouvrage visant à mettre en évidence les différentes perspectives et impacts du processus de professionnalisation. Une première partie analyse ce phénomène du point de vue des espaces de travail et de la formation. Une deuxième partie est consacrée aux défis de la professionnalisation dans l'offre de formation de l'enseignement supérieur. Enfin, une troisième partie aborde la professionnalisation sous l'angle du processus de formation des enseignants et des travailleurs sociaux.

Comme le souligne, dans la préface, Richard Wittorski, spécialiste de la professionnalisation, les responsables de cette édition ont fait le choix très pertinent de sonder différentes cultures professionnelles concernées par ce phénomène. Le milieu des enseignants, ou encore des travailleurs sociaux et des ingénieurs, est confronté à des réalités et des enjeux spécifiques du mouvement de professionnalisation. Chaque contribution s'évertue ainsi 
à définir très clairement ce que la professionnalisation signifie pour son milieu professionnel, quels en sont les impacts et ce qui pourrait constituer des pistes d'ajustement.

La première contribution, de Patricia Champy-Remoussenard, relève l'importance excessive attribuée au mouvement de professionnalisation dans le monde de la formation. La tendance marque une injonction sociétale à toujours rendre les individus plus flexibles et adaptables au monde du travail. Pour faire face à ce processus contraignant et mieux outiller les acteurs de la formation, l'auteure témoigne de l'intérêt de partir des recherches sur l'activité et sur les mutations du travail.

Philippe Mauban et Lucie Roger s'intéressent, de leur côté, aux conséquences de la professionnalisation sur les métiers de la formation, au Québec et en France. Sur ces deux territoires, les auteurs s'inquiètent des risques de disparition des pratiques d'éducation populaire au profit des missions d'employabilité des individus sur le marché de l'emploi. Si la logique économique conduit à une perte des valeurs humanistes de l'éducation, le rapprochement des logiques gestionnaire et pédagogique peut toutefois s'envisager dans certaines conditions. C'est à titre de directeur de service de formation que Marc Poncin défend ce rapprochement dans un texte qui clôt la première partie de l'ouvrage. Il souligne les liens qui rassemblent, depuis longtemps, professionnalisation et formation. Il invite à repenser une conception nouvelle de l'offre de formation en opérant une distinction entre professionnalisation et développement professionnel.

L'ouvrage se poursuit dans cet état d'esprit consistant à mesurer les dangers inhérents au processus de professionnalisation pour mieux en dépasser les contradictions. Abordant le territoire hétérogène de l'enseignement supérieur, Michel Sonntag fait part de son expérience comme chercheur et enseignant dans une école d'ingénieurs. Il s'applique d'abord à décrire en quoi la professionnalisation de l'enseignement supérieur transforme le rapport au savoir en troquant, par exemple, la mise en avant des connaissances contre celle des compétences. L'auteur plaide pour les atouts et les bienfaits d'une solide formation théorique des étudiants. Il complète sa démonstration en s'appuyant sur l'exemple des formations en alternance proposées à des étudiants-ingénieurs en génie mécanique. Dans cette expérience, les tensions et ruptures possibles entre formation académique et pratique sont dépassées par un dispositif de formation garant de l'individualisation des parcours.

Vanessa Boléguin interroge à son tour les bien-fondés de la professionnalisation de l'offre de formation de l'enseignement supérieur, en s'appuyant sur la théorie de la segmentation du marché du travail. Elle soutient que le rapprochement entre des contenus d'enseignement et les compétences requises sur un marché d'emploi peut s'avérer contre-productif. Ce mouvement risque non seulement de générer une dévalorisation du diplôme universitaire généraliste, mais conduit surtout à une dévalorisation de l'individu lui-même, engagé dans une quête, impossible à satisfaire, de qualifications et de reconnaissance. Cette perspective bien sombre est nuancée par la contribution suivante, celle d'Annie Cheminat. Lancienne rectrice et vice-présidente d'université inventorie l'ensemble des offres de diplômes généralistes ou professionnalisants de l'enseignement supérieur français, depuis 
les années 60. La diversité des parcours de formation proposés témoigne d'un mouvement de professionnalisation engagé depuis longtemps dans le système universitaire, avec des offres et des modèles variés.

\section{Vers un autre type de discours et de pensée}

Les écueils et dangers de la professionnalisation semblent surtout s'inscrire dans les modes d'application de ce processus. Finalement, une "professionnalisation à la lettre ", entendue au sens strict, est-elle souhaitable ? Jean-François Marcel relève le défi d'explorer cette question avec une contribution originale rédigée sur le mode du récit épistolaire. Cette liberté dans la forme et dans le ton reste étayée par une argumentation rigoureuse et constitue une autre façon de dépasser les discours convenus sur la professionnalisation. La contribution débouche par ailleurs sur des propositions en faveur de la mise en place d'une professionnalisation "alternative " des enseignants. La hausse du niveau de recrutement des enseignants, qui est une des conséquences de la professionnalisation en cours, devrait notamment s'accompagner d'une plus grande reconnaissance du métier d'enseignant. Françoise Laspeyre pointe également les remédiations souhaitables dans la professionnalisation des futurs enseignants. Pour cette enseignante à l'École supérieure du professorat et de l'éducation (ESPE), la démarche de professionnalisation doit s'ajuster, avec notamment plus de moyens et de temps. Les dernières recherches en sciences de l'éducation peuvent par ailleurs apporter une contribution significative à la formation des futurs enseignants.

On retiendra finalement que le mouvement de professionnalisation peut potentiellement se bonifier avec l'adoption de valeurs plus humanistes que technicistes au cour de ce processus. C'est toute la différence qui sous-tend, par exemple, la notion de métier face au concept d'emploi. C'est ce que soutiennent, tour à tour, Christine Mias et Dominique Besnard dans la professionnalisation des acteurs de la sphère du travail social. Un renouvellement des pratiques de professionnalisation devrait s'envisager, en s'appuyant sur l'activité réelle du travail social qui s'ancre dans la relation humaine, l'aide et l'accompagnement.

\section{Conclusion}

Pour clore cet ouvrage, Stephane Guillon rappelle que le mouvement de professionnalisation actuel participe d'une critique générale de notre système éducatif, considéré comme inadapté aux besoins des entreprises. Au cœur des enjeux de la professionnalisation se retrouvent la relation que nous entretenons au travail et la valeur que nous attribuons à l'éducation dans notre société. 
La force de cet opus est de partir d'une mise au point critique de cette notion polysémique pour nous amener bien au-delà d'une revue des principales orientations disponibles sur la question de la professionnalisation. Parce qu'il pénètre au cœur des mécanismes de la professionnalisation et en extrait les nuances intentionnelles, cet ouvrage pourra sans doute convenir aussi bien aux adeptes quaux opposants du courant de la professionnalisation. Il ne s'agit pas de diaboliser ou de sacraliser ce mouvement, mais bien d'allier l'analyse scientifique aux propositions concrètes d'actions d'amélioration.

\section{Référence de l'ouvrage}

Mohib N. \& Guillon S. (dir.) (2018), La professionnalisation en débat : entre intentions et réalisations, Bruxelles, Peter Lang. 